\title{
Prediction models in urology
}

\author{
Peter T Scardino
}

Predicting risk is an inherent feature of clinical practice. How likely is a man with a given PSA level to have prostate cancer? Can lymph node dissection be safely avoided in a patient with testicular cancer? Should chemotherapy be given after cystectomy for bladder cancer? Traditionally, physicians use clinical judgment to estimate risks, relying on rules of thumb (heuristics) or assigning patients to simple risk groups. In the March issue of Nature Clinical Practice Urology, Novara et al. described a series of nomograms (or models) that can be used to predict whether a man with penile cancer has lymph node metastases and the likelihood that he will survive his cancer for 5 years (Novara et al. [2007] Nat Clin Pract Urol 4: 140-146). Risk stratification schemes have already been published that classify such patients into risk groups, and guidelines are available for the management of men with penile cancer. So why do we need nomograms?

Nomograms are graphical representations of mathematical algorithms that incorporate multiple prognostic factors into optimized statistical models to predict a particular outcome. Since the first report of a nomogram for a urological disease (Kattan MW et al. [1998] J Natl Cancer Inst 90: 766-771), these models have come into widespread use, made easier to use in digital formats (www.nomograms.org). Despite their complexity, nomograms routinely provide more accurate predictions than risk group assignment or expert judgment (Kattan MW [2005] Nat Clin Pract Urol 2: 183-190).

Risk groups are popular but their accuracy is limited, in that they combine individuals with similar but not identical risk factors into a single group and categorize variables, such as PSA $(0-4 \mathrm{ng} / \mathrm{ml}, 4.1-10 \mathrm{ng} / \mathrm{ml}$, etc.), which give more information when analyzed as continuous variables. D'Amico's 'low-risk'

\section{Despite their complexity, nomograms routinely provide more accurate predictions than risk group assignment}

PT Scardino is Editorin-Chief of Nature Clinical Practice Urology.

\section{Competing interests \\ The author declared he has no competing interests}

www.nature.com/clinicalpractice doi:10.1038/ncpuro0776 prognostic group of prostate cancer patients (Stage T1c or T2a, Gleason score <7, PSA level $<10 \mathrm{ng} / \mathrm{ml}$ ) includes patients with a uniformly favorable prognosis, but many patients in his intermediate-risk and high-risk groups actually have an excellent prognosis as well (Kattan MW [2003] Curr Opin Urol 12: 111-116). Erroneous assignment to a high-risk group might lead to an inappropriate treatment decision, such as the pessimistic recommendation to avoid radical prostatectomy in a patient with a Gleason score 8, clinical stage T1c prostate cancer, whose PSA level is $4.5 \mathrm{ng} / \mathrm{ml}$. This 'high-risk' patient actually has a $65 \%$ probability of freedom from biochemical recurrence 10 years after surgery alone (Stephenson et al. [2005] J Clin Oncol 23: 7005-7012).

Nomograms sometimes seem counterintuitive, especially when they include prognostic factors that have not been found to be statistically significant as independent predictors of outcome in multivariable analyses or if they weigh the values of a risk factor nonlinearly. Nevertheless, the prediction is usually more accurate than if fewer factors are included or the results are weighted linearly.

Novara et al. have made a valuable contribution by refining the accuracy of predicting nodal stage and survival in men with penile cancer. These nomograms, which incorporate standard, readily available, clinical and pathological variables are remarkably accurate (Ficarra V et al. [2006] J Urol 175: 1700-1705; Kattan MW et al. [2006] J Urol 175: 21032108). Before nomograms can be accepted in practice, or used to stratify patients in clinical trials, they should be validated in large, independent cohorts. Such nomograms provide patients with what they surely wish for: to know their own prognosis, not that of a risk group to which they might be assigned. 